\title{
Myocardial perfusion scintigraphy and cost effectiveness of diagnosis and management of coronary heart disease
}

\author{
S R Underwood, L J Shaw
}

Heart 2004;90(Suppl V):v34-v36. doi: 10.1136/hrt.2003.019133

$M$ yocardial perfusion scintigraphy (MPS) is a well validated, non-invasive imaging technique that has a valuable role in the diagnosis, management, and assessment of prognosis of coronary heart disease (CHD). The diagnostic accuracy of MPS allows reliable risk stratification and guides the selection of patients for further interventions, such as revascularisation. MPS also has particular advantages over alternative techniques in the management of a number of patient subgroups, including women, the elderly, and those with diabetes. Increased use of MPS will improve patient outcomes and be associated with greater cost effectiveness of treatment, in terms of life-years saved, particularly in these special patient groups. Currently, however, access to MPS in the UK is limited, with inappropriately long waiting times, and MPS activity levels fall short of estimated need.

\section{COST EFFECTIVENESS OF MYOCARDIAL PERFUSION SCINTIGRAPHY}

Principles of cost effectiveness

Several principles underlie why a more accurate diagnostic test with additional prognostic information, such as MPS, can be more cost effective even if it is more expensive than an alternative test such as the exercise ECG (table 1). ${ }^{1}$

For example, fig 1 shows that when a patient with presenting likelihood of CHD of $50 \%$ has an abnormal exercise ECG the post-test likelihood of disease is $73 \%$, which is not sufficiently high to be confident of the diagnosis. A subsequent abnormal MPS gives a likelihood of $96 \%$, but if the same patient had gone directly to MPS the post-test likelihood would have been $90 \%$, which should be sufficiently high to diagnose the presence of CHD depending upon the clinical circumstances.

\section{Costs}

True costs, reflecting consumption of resources, are difficult to estimate. Some analyses of cost effectiveness have used prices as a surrogate for cost, which may be valid from the perspective of the referring clinician, but are less helpful from the perspective of the National Health Service. Two studies have estimated cost, one using UK figures ${ }^{2}$ and the other US

Table 1 Principles of cost effective diagnosis and management of coronary heart disease using myocardial perfusion scintigraphy

- High sensitivity excludes disease more accurately and avoids the need for a secondary test if a less accurate primary test is used.

- High sensitivity leads to fewer false negative tests and avoids the cost of future events in undiagnosed patients with disease

- High specificity reduces the number of false positive tests and consequent downstream testing

- Additional prognostic information avoids the need for further prognostic testing and focuses high cost interventional care on patients with advanced disease and with most to gain in terms of clinical outcome

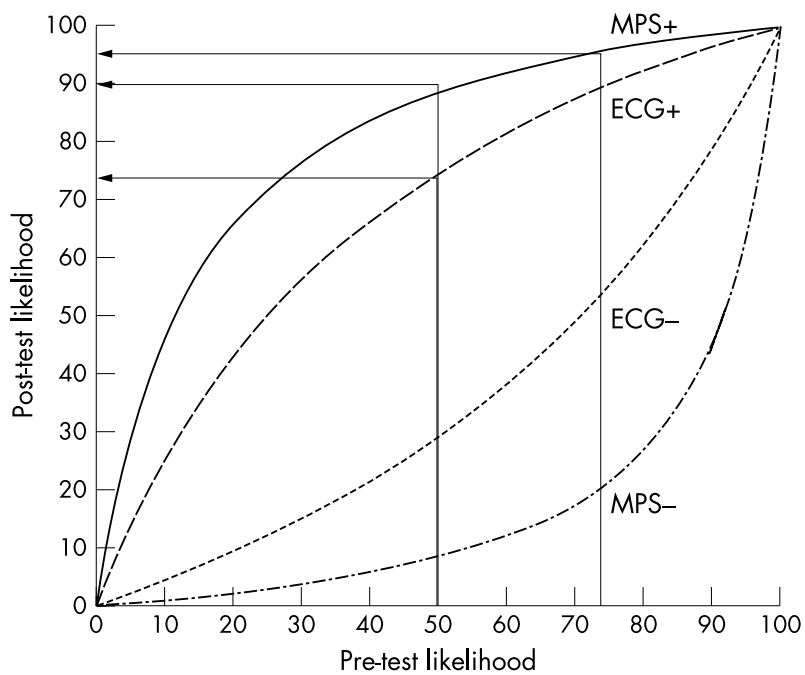

Figure 1 Pre-and post-test likelihood of CHD calculated using Bayesian principles for the exercise ECG and MPS, using sensitivities of $68 \%$ and $92 \%$, respectively, and specificities of $77 \%$ and $88 \%$, respectively. The curved lines from top to bottom represent MPS+, ECG+, ECG- and MPS-.

figures, ${ }^{3}$ but the findings are similar at $£ 220$ in the UK and $£ 179$ (range $£ 159-348$ ) in the USA (table 2). These costings are sensitive to throughput. A reasonable throughput per camera is 2000 patients per year, but this can be increased to as much as 4000 in high volume centres by running the camera 12 hours per day and six days per week.

\section{Cost effectiveness of MPS}

Although randomised controlled and blinded trials are often used to evaluate the cost effectiveness of treatment, this study design is difficult or impossible in the case of diagnostic testing. However, some data have been derived from decision analytical models and these have demonstrated the cost effectiveness of MPS both in patients presenting with stable chest pain syndromes ${ }^{5}$ and in pre-operative risk assessment. ${ }^{6}$ Garber and colleagues showed that in 55 year old men presenting with chest pain, a strategy of MPS proceeding to angiography compared with exercise ECG proceeding to angiography cost $£ 25000$ per quality adjusted life year (QALY), ${ }^{7}$ a figure that is generally regarded as acceptable. ${ }^{8}$ The benefit of MPS would have been even greater if a more realistic model had been used, since it was assumed that all patients with disease proceed to angiography, whereas, as demonstrated above, it is possible to treat low risk patients with CHD without the need for angiography. Patterson and colleagues also found initial testing with MPS to be more cost

Abbreviations: CHD, coronary heart disease; END, economics of noninvasive diagnosis; MPS, myocardial perfusion scintigraphy; QALY, quality adjusted life year 
Table 2 Cost of common diagnostic tests calculated using principles that estimate the true consumption of resources. ${ }^{2}{ }^{3}$ Figures for stress echocardiography are estimated from Medicare charges ${ }^{4}$

\begin{tabular}{|c|c|c|c|}
\hline & EMPIRE $^{2}$ & USA average $^{3}$ & USA range $^{3}$ \\
\hline Rest ECG & $£ 20$ & & \\
\hline Exercise ECG & $£ 70$ & & \\
\hline Rest echocardiography & $£ 100$ & $£ 55$ & $£ 39-£ 207$ \\
\hline Stress echocardiography & & $£ 164^{4}$ & $£ 114-£ 213^{4}$ \\
\hline Computed $x$ ray tomography & & $£ 172$ & $£ 55-£ 288$ \\
\hline MPS & $£ 220$ & $£ 179$ & $£ 159-£ 348$ \\
\hline MRI & & $£ 529$ & $£ 318-£ 739$ \\
\hline PET & & $£ 771$ & $£ 582-£ 891$ \\
\hline Coronary angiography & $£ 1100$ & $£ 1097$ & $£ 516-£ 2873$ \\
\hline
\end{tabular}

EMPIRE, economics of myocardial perfusion imaging in Europe; MRI, magnetic resonance imaging; MPS, myocardial perfusion scintigraphy; PET, positron emission tomography.

effective than the exercise ECG, although again the model assumed that all patients with disease undergo angiography. ${ }^{9}$ Kuntz and colleagues found a higher incremental cost effectiveness of initial MPS over the exercise ECG of $£ 34250$ in a 55 year old man with atypical chest pain, but they used an unrealistically low specificity of $64 \%$ for MPS and again assumed that all patients with disease undergo angiography. ${ }^{4}$ It would be valuable to repeat these models assuming that patients with abnormal but low risk noninvasive tests are treated medically in the first instance, since the prognostic power of MPS is likely to lead to even greater cost effectiveness.

Interpretation of these numbers should be placed in the context of other generally accepted medical interventions. For example, coronary artery bypass grafting for patients with triple vessel coronary artery disease and severely impaired left ventricular function costs £25 500 per QALY gained, ${ }^{10}$ and cholesterol lowering therapy in a 60 year old man with cholesterol of $7.5 \mathrm{mmol} / \mathrm{l}$ costs approximately £30 000 per QALY. ${ }^{11}$ Thus, MPS appears to be a cost effective use of resources compared with other generally accepted medical procedures.

Mathematical simulations have the advantage of using data from diverse sources or from expert opinion, but they are limited by a lack of real world effectiveness data. However, two controlled clinical studies have demonstrated

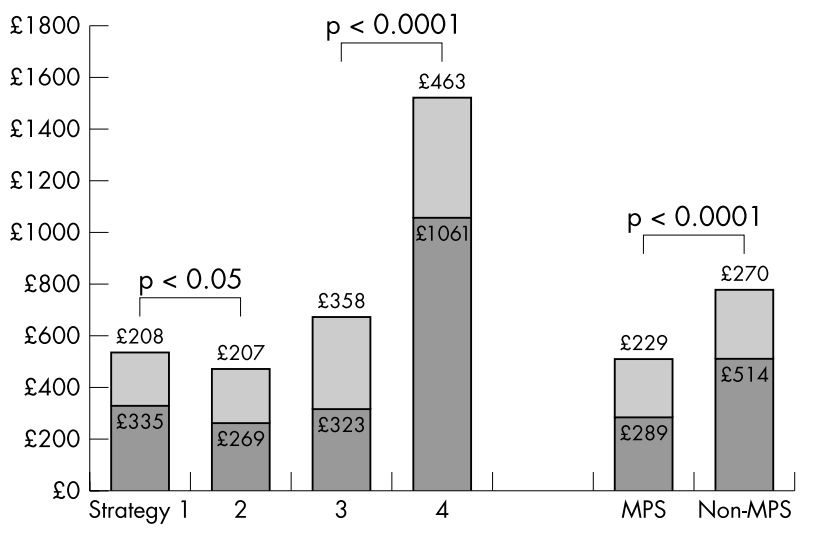

Figure 2 Two year costs of diagnosis (dark grey) and management (light grey) in patients without CHD but presenting with stable chest pain syndromes, according to strategy of investigation and type of hospital. The strategies of investigation are: (1) exECG-angio; (2) exECG-MPSangio; (3) MPS-angio; (4) angio. Angio, angiography; exECG, exercise ECG; MPS, myocardial perfusion scintigraphy. MPS = regular user of MPS; non-MPS = occasional user of MPS. ${ }^{2}$ savings for similar outcomes using MPS in patients presenting with chest pain. The economics of myocardial perfusion imaging in Europe (EMPIRE) study compared patients presenting with stable chest pain syndromes to centres that routinely use MPS and those that do not, in each of four European countries. ${ }^{2}$ Diagnostic strategies using MPS were cheaper and equally effective than those that did not, both for the costs of diagnosis and for overall two year management costs, but patient outcome was the same (fig 2).

The economics of non-invasive diagnosis (END) study was a larger (11 372 patients) registry study with very similar findings of $30-40 \%$ savings in costs over 2.5 years in patients with stable chest pain syndromes undergoing initial MPS and selective angiography (fig 3). ${ }^{12}$ An additional series of 9521 patients showed that MPS was more cost effective than stress echocardiography in patients with known CHD and stable chest pain at \$39347 per life-year saved. ${ }^{13}$ Unfortunately, modelling studies have provided conflicting results on the comparative cost effectiveness of strategies using MPS and stress echocardiography ${ }^{4}{ }^{14}$ and there are insufficient clinical data to make a firm statement on this.

Other clinical studies indirectly support the cost effectiveness of MPS. In a US practice making routine use of MPS the number of patients with normal MPS proceeding to angiography is $1 \%,{ }^{15}$ whereas in a UK rapid access chest pain clinic that makes little use of MPS, coronary angiography rates are high and $56 \%$ of coronary angiograms in women are normal. ${ }^{16}$ It has been estimated that routine use

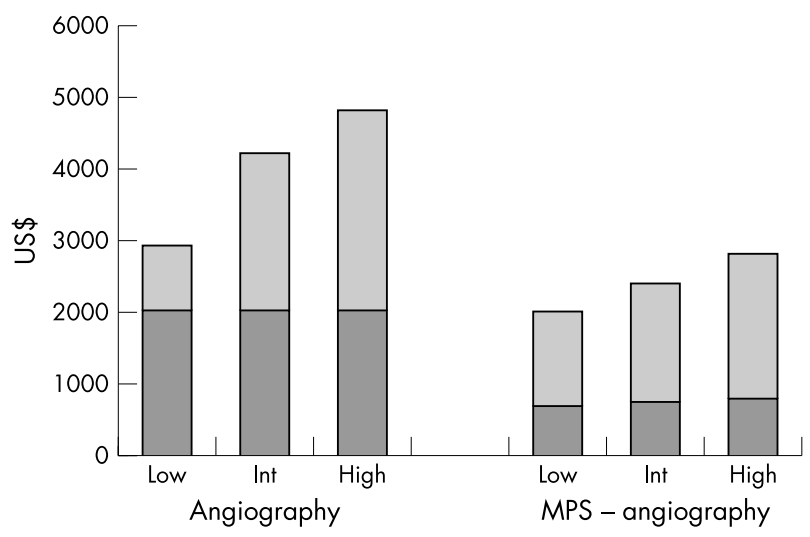

Figure 3 Two and a half year costs of diagnosis (dark grey) and management (light grey) in 11372 patients presenting with stable chest pain syndromes who underwent initial angiography or MPS with selective angiography, according to low, intermediate or high pre-test likelihood of CHD. ${ }^{12}$ 
of MPS in this setting would reduce cost by between $£ 30000$ and $£ 65000$ per year, mainly by reducing the normal coronary angiography rate. ${ }^{17}$ Similarly in the USA it has been estimated that about one third of referrals to coronary angiography are inappropriate. ${ }^{18}$

\section{Clinical outcomes}

Modelling studies indicate that clinical outcome is improved by MPS at a cost per QALY that is acceptable and that life expectancy is increased by between seven days and two years. ${ }^{6}$ Because of the scale and nature of the studies that would be required, it has been more difficult to confirm this finding in clinical studies. However, in a reanalysis of the END data, ${ }^{12}$ Shaw and colleagues have shown that testing with initial MPS adds between one and two years of life, ${ }^{19}$ and in a separate study they showed that MPS added 0.5 years compared with stress echocardiography. ${ }^{13}$

\section{CONCLUSION}

MPS is a well established, non-invasive imaging technique with a large body of evidence to support its effectiveness in the diagnosis and management of angina and myocardial infarction. It is more accurate than the exercise ECG in detecting myocardial ischaemia and it is the single most powerful technique for predicting future coronary events.

The high diagnostic accuracy of MPS allows reliable risk stratification and guides the selection of patients for further interventions, such as revascularisation. This in turn allows more appropriate utilisation of resources, with the potential for both improved clinical outcomes and greater cost effectiveness. Evidence from modelling and observational studies supports the enhanced cost effectiveness associated with MPS use. In patients presenting with stable or acute chest pain, strategies of investigation involving MPS are more cost effective than those not using the technique. MPS also has particular advantages over alternative techniques in the management of a number of patient subgroups, including women, the elderly, and those with diabetes, and its use will have a favourable impact on cost effectiveness in these groups.

\section{ACKNOWLEDGEMENTS}

This submission is based upon the deliberations of a consensus conference on the evidence for the clinical and cost effectiveness of MPS, organised by the British Nuclear Cardiology Society in January 2003. Members of the writing group were C Anagnostopoulos (cochair), M Cerqueira, PJ Ell, J Flint, M Harbinson, A Kelion, A Al Mohammad, EM Prvulovich, LJ Shaw, AC Tweddel, SR Underwood (co-chair)

\footnotetext{
Authors' affiliations

Authors' affiliations
S R Underwood, Imperial College, Royal Brompton Hospital, London, UK
}

L J Shaw, Atlanta Cardiovascular Research Institute, Atlanta, Georgia, USA

Correspondence to: Professor S Richard Underwood, Imperial College London, Royal Brompton Hospital, Sydney Street, London SW3 6NP,

UK; r.underwood@imperial.ac.uk

\section{REFERENCES}

1 Shaw LJ, Eisenstein EL, Hachamovitch R, et al. A primer of biostatistic and economic methods for diagnostic and prognostic modeling in nuclear cardiology: part II. J Nucl Cardiol 1997;4:52-60.

2 Underwood SR, Godman B, Salyani S, et al. Economics of myocardial perfusion imaging in Europe-the EMPIRE study. Eur Heart J 1999;20:157-66.

3 Mark DB, Shaw $\sqcup$, Laver MS, et al. Is atherosclerotic imaging cost effective? J Am Coll Cardiol 2003:41:1906-17.

4 Kuntz KM. Cost-effectiveness of diagnostic strategies for patients with chest pain. Ann Intern Med 1999;130:709-18.

5 Maddahi J, Ghambir SS. Cost-effective selection of patients for coronary angiography. J Nucl Cardiol 1997;4:S141-51.

6 Shaw L, Hachamovitch R, Eisenstein E, et al. Cost implications for implementing a selective preoperative risk screening approach for peripheral vascular surgery patients. Am J Managed Care 1997;3:1817-27.

7 Garber AM, Solomon NA. Cost-effectiveness of alternative test strategies for the diagnosis of coronary artery disease. Ann Intern Med 1999;130:719-28.

8 Goldman L, Garber AM, Grover SA, et al. 27th Bethesda Conference: matching the intensity of risk factor management with the hazard for CAD events. Task force 6: cost effectiveness of assessment and management of risk factors, J Am Coll Cardiol 1996;27:1020-30.

9 Patterson RE, Eng C, Horowitz SF, et al. Bayesian comparison of costeffectiveness of different clinical approaches to coronary artery disease. J Am Coll Cardiol 1984:4:278-89.

10 Wong JB, Sonnenberg FA, Salem DN, et al. Myocardial revascularization for chronic stable angina. Analysis of the role of percutaneous transluminal coronary angioplasty based on data available in 1989. Ann Intern Med 1990;113:852-71.

11 Goldman L, Weinstein MC, Goldman PA, et al. Cost-effectiveness of HMGCoA reductase inhibition for primary and secondary prevention of coronary heart disease. JAMA 1991;265:1145-51.

12 Shaw LJ, Hachamovitch R, Berman DS, et al. The economic consequences of available diagnostic and prognostic strategies for the evaluation of stable angina patients: an observational assessment of the value of precatheterization ischemia. Economics of noninvasive diagnosis (END) multicenter study group. J Am Coll Cardiol 1999;33:661-9.

13 Shaw L, Marwick TH, Sawada S, et al. Prognostic value and cost effectiveness of exercise-induced ischemia by echocardiography versus SPECT imaging for the evaluation of patients with stable chest pain [abstract]. Circulation 2002;106(suppl II):II-610.

14 Lee DS, Jang MJ, Cheon GJ, et al. Comparison of the cost-effectiveness of stress myocardial perfusion SPECT and stress echocardiography in suspected coronary artery disease considering the prognostic value of false-negative results. J Nucl Cardiol 2002;9:515-22.

15 Bateman TM. Nuclear cardiology in private practice. J Nucl Cardiol 1997;4:S184-8.

16 Wong $Y$, Rodwell A, Dawkins S, et al. Sex differences in investigation results and treatment in subjects referred for investigation of chest pain. Heart 2001;85:149-52.

17 Underwood SR. Cost-effective investigation of chest pain (electronic correspondence). Heart 2001 http://heart.bmijournals.com/cgi/eletters/ $85 / 2 / 149$.

18 Scanlon PJ, Faxon DP, Audet AM, et al. ACC/AHA guidelines for coronary angiography. J Am Coll Cardiol 1999;33:1756-824.

19 Shaw L, Hachamovitch R, Papatheofanis FJ. Outcomes and technology assessment in nuclear medicine. Reston, Virginia: Society of Nuclear Medicine Press, 1999. 